\title{
Association of HLA-B27 and its subtypes with ankylosing spondylitis and clinical manifestations of ankylosing spondylitis in different HLA-B27 subtypes: comment on the article by Lin et al.
}

\author{
Sasan Fallahi ${ }^{1,2}$
}

Received: 12 June 2017 / Accepted: 8 August 2017 / Published online: 21 August 2017

(c) Springer-Verlag GmbH Germany 2017

Keywords Ankylosing spondylitis · HLA-B27 . Spondyloarthropathies $\cdot$ Symptom assessment

To the Editor,

I read the interesting manuscript by Lin et al. which provided a review on association of HLA-B27 with ankylosing spondylitis (AS) and clinical features of AS in different HLAB27 subtypes [1]. I hereby draw your attention that some relevant articles have been missed from this review, and consequently, the results may be affected [2-4]. For example, current meta-analysis demonstrated the association of AS with HLA-B27, HLA-B27*02, and HLA-B27*04. However, in a study on 163 patients with AS in Iran, HLA-B27*05 and then HLA-B27*02 were the most prevalent HLA-B*27 subtypes. Conversely, HLA-B7*04 and HLA-B*2707 were rarely seen [2]. Moreover, literature review indicates that HLA-B27 subtypes are heterogeneous in various races and ethnicities entire the world. Therefore, it is logical that clinical features of AS associated with HLA-B27 subtypes are dissimilar in various parts of the world with different races

This comment refers to the article available at doi:10.1007/ s00296-017-3741-2.

An author's reply to this comment is available at doi:10.1007/ s00296-017-3789-z.

Sasan Fallahi

sfallahi@tums.ac.ir; drsfallahi@gmail.com

1 Rheumatologist, Internal Medicine Division, Baharloo Hospital, Tehran University of Medical Sciences, Behdari Street, South Kargar Street, Tehran 1339973111, Iran

2 Rheumatology Research Center, Shariati Hospital, Tehran University of Medical Sciences, Tehran, Iran and ethnicities. Therefore, the results of this analysis should not be generalized to all patients with AS. Clinical features of patients with AS with different HLA-B27 subtypes are suggested to be reviewed and meta-analyzed separately in various races and parts of the world. Research reviews on different races and regions of the world individually (like Europe, Middle East, Far East, and Africa) may help to exploring more exactly the association between symptoms and HLA-B27 subtypes in AS disease.

Compliance with ethical standards

Conflict of interest The author declares that he has no conflict of interest to report.

\section{References}

1. Lin H, Gong YZ (2017) Association of HLA-B27 with ankylosing spondylitis and clinical features of the HLA-B27 associated ankylosing spondylitis: a meta-analysis. Rheumatol Int. doi:10.1007/ s00296-017-3741-2 (Epub ahead of print)

2. Fallahi S, Mahmoudi M, Nicknam MH, Gharibdoost F, Farhadi E, Saei A, Nourijelyani K, Ahmadzadeh N, Jamshidi AR (2013) Effect of HLA-B*27 and its subtypes on clinical manifestations and severity of ankylosing spondylitis in Iranian patients. Iran J Allergy Asthma Immunol 12(4):321-330

3. Mou Y, Zhang P, Li Q, Lin Z, Liao Z, Wei Q, Gu J (2015) Clinical features in juvenile-onset ankylosing spondylitis patients carrying different B27 subtypes. Biomed Res Int. doi:10.1155/2015/594878

4. Omair MA, AlDuraibi FK, Bedaiwi MK, Abdulaziz S, Husain W, El Dessougi M, Alhumaidan H, Al Khabbaz HJ, Alahmadi I, Omair MA, Al Saleh S, Alismael K, Al Awwami M (2017) Prevalence of HLA-B27 in the general population and in patients with axial spondyloarthritis in Saudi Arabia. Clin Rheumatol. doi:10.1007/s10067-017-3655-0 (Epub ahead of print) 